\title{
Lehrerfortbildung Mathematik - Konzepte und Wirkungsforschung - Editorial
}

\author{
Rolf Biehler $\cdot$ Petra Scherer
}

Online publiziert: 6 . November 2015

(C) GDM 2015

Lehrerfortbildung als dritte Phase der Lehrerbildung ist für die Didaktik der Mathematik sowohl eine praktische wie eine theoretische Herausforderung. Die Fachdidaktik hat sich immer schon praktisch an Lehrerfortbildungen beteiligt und diese auch initiiert und theoretisch konzipiert. Die theoretische Herausforderung bezieht sich einerseits auf die Entwicklung von nachhaltig wirksamen Konzepten für Lehrerfortbildung auf der Basis von Forschungen sowie auf die Konzipierung von Wirkungsforschungen (vgl. Rösken-Winter et al. 2015).

Nicht zuletzt in der Folge der Ergebnisse von TIMSS und PISA hat die Lehrerfortbildung in Deutschland eine Aufwertung erfahren. In der Erziehungswissenschaft und der Mathematikdidaktik haben sich die Forschungen zur Lehrerfortbildung wesentlich ausgeweitet (vgl. z. B. Lipowsky 2010; Rösken 2011), aber auch große Forschungsdefizite und -desiderata identifiziert.

Empirische Studien zeigen, dass die fachbezogenen Facetten der professionellen Kompetenz von Mathematiklehrkräften eng zusammenhängen (Blömeke und Delaney 2012; Schilling et al. 2007; Krauss et al. 2008) wie auch das mathematikdidaktische Wissen besonders hoch mit den im Mathematikunterricht erreichten Schülerleistungen korreliert (Baumert et al. 2010; Kunter et al. 2013). Diese Ergebnisse unterstützen die Anstrengungen für den Lehrerfortbildungsbereich. Die Innovation über neu eingestellte Lehrkräfte ist insgesamt auch unter mittelfristiger Perspektive zu wenig wirksam.

\footnotetext{
R. Biehler $(\bowtie)$

Institut für Mathematik, Universität Paderborn,

Warburger Str. 100,

33098 Paderborn, Deutschland

E-Mail: biehler@math.upb.de

P. Scherer

Fakultät für Mathematik, Universität Duisburg-Essen, Campus Essen,

45127 Essen, Deutschland

E-Mail: petra.scherer@uni-due.de
} 
Die Deutsche Telekom Stiftung hatte mit dem Projekt „Mathematik Anders Machen“" ab 2007 neue Akzente in der Förderung der Fortbildung von Mathematiklehrkräften gesetzt, die letztlich in die Förderung des Deutschen Zentrums für Lehrerbildung Mathematik (DZLM) ab dem Jahre 2011 mündeten, das einen länderübergreifenden, systematischen und forschungsbasierten Zugang zur Lehrerfortbildung verfolgt und die Vernetzung der Lehrerfortbildungsaktivitäten in Deutschland fördern soll (www.dzlm.de; DZLM 2014, 2015).

Die Initiative zu diesem Themenschwerpunkt haben wir als JMD-Herausgebende und zugleich im DZLM tätige Personen ergriffen und Wissenschaftlerinnen und Wissenschaftler außerhalb und innerhalb des DZLM gebeten, themenbezogene Manuskripte einzureichen, um verschiedene Richtungen und Forschungsansätze zu repräsentieren.

Die vorliegenden Beiträge reichen von historischen Aufarbeitungen der Entwicklung der Lehrerbildung (Beitrag Törner) über konzeptionelle Überlegungen zur Auswahl adäquater Inhalte der Lehrerfortbildung (Beitrag Prediger et al.) und zur fortbildungsmethodischen Gestaltung von Lehrerfortbildungen (Beitrag Barzel und Selter) bis hin zu Wirkungsforschungen zu konkreten Fortbildungsmaßnahmen, wobei hier fachdidaktische Fortbildungsthemen im Vordergrund stehen wie die prozessbezogene Kompetenz des Problemlösens (Beitrag Besser et al.) und die inhaltsbezogene Kompetenz des Umgangs mit dem Funktionsbegriff (Beitrag Busch et al.).

Die Beiträge ordnen sich damit in aktuelle Forschungslinien der Lehrerfortbildungsforschung ein:

- Bedingungsfaktoren und Gelingensfaktoren von Lehrerbildung in Mathematik

- Modelle für Lehrerkompetenzen

- Konzepte und Programme von Lehrerfortbildung

- Gestaltungsprinzipien für Lehrerfortbildungen

- Wirkungen von Lehrerfortbildungen (Einstellung und Kompetenzen von Fortgebildeten, Wirkung auf deren Lehr- bzw. Fortbildungspraxis)

Günter Törners Beitrag zu ,,Verborgene Bedingungs- und Gelingensfaktoren bei Fortbildungsmaßnahmen in der Lehrerbildung Mathematik - subjektive Erfahrungen aus einer deutschen Perspektive“ arbeitet in historischer Perspektive der Auseinandersetzung mit der Lehrer(fort-)bildung in (West-) Deutschland seit den 1970er Jahren auf, stellt vor diesem Hintergrund Akzente der neueren Bemühungen in der Lehrerfortbildung heraus und stellt den Anschluss an die internationalen Diskussionen und Forschungen her. Dabei werden sowohl normative bildungspolitische Dokumente wie theoretische und empirische Forschungen zur Lehrerbildung einbezogen. Ebenso wird die Rolle der unterschiedlichen Akteure im Feld, von Fachgesellschaften und Bildungsadministration bis zur universitären Mathematik und Mathematikdidaktik, verdeutlicht.

Bei der Konzeption von Fortbildungsmaßnahmen geht es sowohl um die Frage des Inhalts (das Was) als auch um Fragen der methodisch-didaktischen Gestaltung (das Wie). Die nächsten beiden Beiträge diesen Themenhefts befassen sich mit diesen Fragen.

Susanne Prediger, Uta Quasthoff, Anna-Marietha Vogler und Vivien Heller diskutieren in ihrem Beitrag „How to determine what teachers should learn? Five steps for 
content specification of professional development programs, exemplified by ,moves supporting participation in classroom discussions “" Fragen der inhaltlichen Gestaltung von Fortbildungsmaßnahmen. Sie wenden sich gegen eine häufig anzutreffende Beliebigkeit bei der inhaltlichen Spezifizierung von Fortbildungen und stellen dem ein Fünf-Schritte-Programm gegenüber, das theoretisch und empirisch begründet ist und das die forschungsbasierte Generierung von Fortbildungsinhalten unterstützen kann. Die einzelnen Schritte, wie etwa die Frage der Relevanz eines Fortbildungsinhalts aus theoretischer Perspektive (Schritt 1) oder die Spezifizierung der professionellen Anforderungen einer Lehrperson (Schritt 2) und der Exploration der entsprechenden Perspektiven (Schritt 3), jeweils empirisch erhoben, werden am Beispielinhalt ,partizipationsförderliche Impulse in Unterrichtsgesprächen“ illustriert.

Bärbel Barzel und Christoph Selter fokussieren in ihrem Beitrag auf „Die DZLMGestaltungsprinzipien für Fortbildungen“" stärker die methodische Gestaltung von Fortbildungsmaßnahmen im Fach Mathematik. Im Zentrum stehen sechs zentrale Gestaltungsprinzipien für Fortbildungen (Kompetenzorientierung, Teilnehmerorientierung, Lehr-Lern-Vielfalt, Fallbezug, Kooperationsanregung, Reflexionsförderung). Diese basieren auf theoretischen und empirischen Forschungen, sowie auf umfangreichen Erfahrungen zur Fortbildungspraxis in verschiedenen Projekten und werden den Fortbildungsangeboten im DZLM zugrunde gelegt. Zu den einzelnen Prinzipien werden einerseits relevante Forschungsbefunde herangezogen, andererseits erfolgen thematische Konkretisierungen für verschiedene Schulstufen und mathematische Inhalte, um auf diese Weise die allgemeine Relevanz und potentielle Wirksamkeit der Gestaltungsprinzipien zu begründen.

In einem dritten Block finden sich zwei Beiträge zur Wirkungsforschung von Lehrerfortbildungen, und zwar bezogen auf die Einstellungs- und die Kompetenzveränderungen, die sich nach und durch die Fortbildung ergeben haben. Dabei ist zu berücksichtigen, dass ein Kontroll- und Experimentalgruppendesign hinsichtlich der üblichen Rahmenbedingungen, unter denen Lehrerfortbildungen durchgeführt werden, nur schwierig zu realisieren ist.

In ihrem Beitrag „Theoretische Konzeption und empirische Wirkung einer Lehrerfortbildung am Beispiel des mathematischen Problemlösens" verwenden Michael Besser, Dominik Leiss und Werner Blum ein Kontrollgruppendesign, um Effekte auf das fachbezogene und fachdidaktische Wissen bezüglich des Problemlösens im Mathematikunterricht zu erfassen und im Hinblick auf andere Wissensfacetten zu kontrollieren. Damit wird sowohl ein Beitrag zum Design von Lehrerfortbildungsforschung geleistet wie auch ein Beitrag zur Erfassung von Facetten der Lehrerkompetenz.

Julia Busch, Bärbel Barzel und Timo Leuders befassen sich mit der „Entwicklung eines Instruments zur kategorialen Beurteilung der Entwicklung diagnostischer Kompetenzen von Lehrkräften im Bereich Funktionen“" und leisten somit einen neuen Beitrag zur diagnostischen Erfassung von Lehrerkompetenzen. Dies geschieht mit Hilfe von Items, in denen Schülerlösungen durch Lehrkräfte in einem freien Antwortformat diagnostisch eingeordnet werden müssen. Dabei werden die Lehrerantworten kategorial erfasst, so dass eine Kompetenzentwicklung auch für quantitative Methoden zugänglich gemacht werden kann. Das Instrument wird auf eine Lehrerfortbildungsmaßnahme angewendet, zu der der erzielte Kompetenzzuwachs gut her- 
ausgearbeitet werden kann. Damit ist auch eine geeignete Basis für die Anwendung in Kontrollgruppendesigns gelegt.

Die fünf Beiträge zu unserem Themenheft demonstrieren exemplarisch zentrale Facetten und Ebenen der Lehrerbildungsforschung, die auch in Zukunft wichtig bleiben werden. Es war für uns von Bedeutung, nicht nur empirische Arbeiten, sondern auch konzeptionelle Arbeiten und die Entwicklung in der Mathematikdidaktik selbst reflektierende Arbeiten mit aufzunehmen, da wir in der Verbindung und Vernetzung der verschiedenen Forschungsebenen ein hohes Potential sehen.

\author{
Paderborn und Essen \\ im Oktober 2015 \\ Rolf Biehler, Petra Scherer \\ Herausgebende des JMD Themenheftes
}

\title{
Literatur
}

Baumert, J., Kunter, M., Blum, W., Brunner, M., Voss, T., Jordan, A., et al. (2010). Teachers' mathematical knowledge, cognitive activation in the classroom, and student progress. American Educational Research Journal, 47(1), 133-180.

Blömeke, S., \& Delaney, S. (2012). Assessment of teacher knowledge across countries: A review of the state of research. ZDM Mathematics Education, 44(3), 223-247.

DZLM (2014). Theoretischer Rahmen des Deutschen Zentrums für Lehrerbildung Mathematik. http:// www.dzlm.de/files/uploads/DZLM-0.0-Theoretischer-Rahmen-20150218_FINAL-20150324.pdf. Gesehen 11. Oktober 2015.

DZLM (2015). Selbstbericht 2015. Qualifizieren. Forschen. Netzwerke bilden. http://www.dzlm.de/files/ uploads/DZLM-Selbstbericht-2015-Final-für\%20Webseite\%2023-07.pdf. Gesehen 11. Oktober 2015.

Krauss, S., Baumert, J., \& Blum, W. (2008). Secondary mathematics teachers' pedagogical content knowledge and content knowledge: validation of the COACTIV constructs. ZDM Mathematics Education, 40(5), 873-892.

Kunter, M., Baumert, J., Blum, W., Klusmann, U., Krauss, S., \& Neubrand, M. (Hrsg.). (2013). Professionelle Kompetenz von Lehrkräften - Ergebnisse des Forschungsprogramms COACTIV. Münster: Waxmann.

Lipowsky, F. (2010). Lernen im Beruf. Empirische Befunde zur Wirksamkeit von Lehrerfortbildung. In F. Müller, A. Eichenberger, M. Lüders \& J. Mayr (Hrsg.), Lehrerinnen nud Lehrer lernen - Konzepte und Befunde zur Lehrerfortbildung (S. 51-72). Münster: Waxmann.

Rösken, B. (2011). Hidden Dimensions in the Professional Development of Mathematics Teachers - InService Education for and with Teachers. Rotterdam/Boston/Taipeh: Sense Publisher.

Rösken-Winter, B., Hoyles, C., \& Blömeke, S. (2015). Evidence-based CPD: Scaling up sustainable interventions. ZDM Mathematics Education, 47(1), 1-12.

Schilling, S. G., Blunk, M., \& Hill, H. C. (2007). Test Validation and the MKT Measures: Generalizations and Conclusions. Measurement: Interdisciplinary Research and Perspectives, 5(2-3), 118-128. 